\title{
0047 EMS MANAGEMENT SYSTEMS FOR THE TRAFFIC INJURY PATIENTS AT THE SCENE WHEN EVENTS OVERLAP
}

K Wangsri* Correspondence: Srinagarind Hospital, Faculty of Medicine, Khonkaen University, EMS Unit, Accident and Emergency Department, Srinagarind Hospital, Khonkaen 40002, Thailand

\subsection{6/ip.2010.029215.47}

Background The Emergency Medical Service (EMS) at Srinagarind Hospital consists of mobile teams that attend traffic accidents and provide emergency care at the scene. The hospital has decided to train additional members of the nursing staff so that they can be called upon to attend traffic accidents when the EMS teams are already employed elsewhere. This study aims to: ensure that traffic injury patients receive appropriate care at the scene when the EMS teams are already employed elsewhere, by providing selected Accident and Emergency (AE) department nursing staff to provide this care.

Approach Analysis of emergency medical services activities over the past 2 years. Develop protocols on how the AE department should respond when EMS team is unavailable. Provide AE department staff with the knowledge needed to work as an emergency medical services team. Design and implement an appropriate management information system for monitoring and evaluation of $\mathrm{AE}$ department staff activities when working as an EMS team. Evaluate and report the outcomes of this project.

Results The AE department has developed protocols on how to respond to EMS call outs when the regular team is unavailable $-80 \%$ of EMS teams are able to leave the hospital within 2 min of being called out. EMS staff rated their performance at $89.81 \%$ satisfactory. $(n=9)$ - users of EMS services rated the service at $74.07 \%$ satisfactory. $(n=150)$

Recommendation Their must be ongoing staff training and development activities to increase the proportion of teams that can respond to being called out within 2 min. 